\title{
Spatial reasoning skills about 2D representations of 3D geometrical shapes in grades 4 to 9
}

\section{Taro Fujita $^{1}$ (D) $\cdot$ Yutaka Kondo $^{2} \cdot$ Hiroyuki Kumakura $^{3} \cdot$ Susumu Kunimune $^{3}$. Keith Jones ${ }^{4}$ (D)}

Received: 30 October 2019 / Revised: 13 April 2020 / Accepted: 2 May 2020 /

Published online: 27 May 2020

(C) The Author(s) 2020

\begin{abstract}
Given the important role played by students' spatial reasoning skills, in this paper we analyse how students use these skills to solve problems involving 2D representations of 3D geometrical shapes. Using data from in total 1357 grades 4 to 9 students, we examine how they visualise shapes in the given diagrams and make use of properties of shapes to reason. We found that using either spatial visualisation or property-based spatial analytic reasoning is not enough for the problems that required more than one step of reasoning, but also that these two skills have to be harmonised by domainspecific knowledge in order to overcome the perceptual appearance (or "look") of the given diagram. We argue that more opportunities might be given to both primary and secondary school students in which they can exercise not only their spatial reasoning skills but also consolidate and use their existing domain-specific knowledge of geometry for productive reasoning in geometry.
\end{abstract}

Keywords Spatial reasoning $\cdot$ Domain-specific knowledge of geometrical shapes $\cdot 2 \mathrm{D}$ representations $\cdot 3 \mathrm{D}$ shapes

\section{Introduction}

In a recent research review, Jones and Tzekaki (2016) summarise how research is focussing on 'the development of students' knowledge regarding understanding of

Taro Fujita

t.fujita@exeter.ac.uk

1 University of Exeter, 79 Heavitree Rd, Exeter EX1 2LU, UK

2 Nara University of Education, Nara, Japan

3 Shizuoka University, Shizuoka, Japan

4 University of Southampton, Southampton, England 
geometric figure, definitions and inclusion relations, identification of shapes and language issues" (p. 109). In this, geometrical reasoning is a vital component in students' tackling of problems involving geometrical figures. In geometry, figures are often in the form of diagrams (on paper or computer screen) and working with such representations (including geometric figures imagined by students) is important during geometric reasoning (Lowrie 2012; Fujita et al. 2017). When working with problems involving 2D representations of 3D shapes, students use various skills to solve the problems. Mulligan et al. (2018) say that an "integrated definition of spatial reasoning" includes the skills related to mental manipulations of visual stimuli, transforming spatial forms into other visual forms with analytical thinking of structural features of spatial forms (p. 78). At the same time, research studies also suggest that spatial skills such as manipulating given representations and reasoning can be improved through certain training or interventions (e.g. Fischbein 1993, p. 156; Lowrie et al. 2019).

Our goal is to identify students' spatial reasoning skills, and how they use their skills and knowledge to solve geometrical problems, in order to design interventions to improve teaching. In this paper, we particularly focus on the following two research questions:

- RQ1: How do students across the grades use their spatial reasoning skills and domain-specific knowledge when tackling problems involving 3D geometrical shapes represented in 2D?

- RQ2: What can be learnt from students' use of such skills and knowledge in order to design and implement future interventions to improve their skills and knowledge?

We pose these questions because we speculate students' use of spatial reasoning skills might be related to their domain-specific knowledge around elements, properties and concepts of geometrical figures (see next section). We study students from across the grades from primary to secondary because in mathematics education research not so much has been reported about students' reasoning with 3D shapes across primary and secondary schools. More research studies are needed, not only of the teaching and learning of 3D geometry in primary schools (Sinclair and Bruce 2015; Sinclair et al. 2016) but also across primary to secondary schools to obtain a more comprehensive picture of the development of students' understanding. In particular, if common patterns in student skills and difficulties can be identified, then such information is likely to be useful for designing effective teaching interventions for improving students' spatial skills.

In what follows, we first construct our theoretical framework to consider the relationships between, and student use of, spatial reasoning skills and domainspecific knowledge of geometry. We then report findings from our survey of 1357 students from grades 4-9 in Japan and our observations of students' thinking in a classroom-based study with a lesson based on a sample of tasks used in the survey. We then discuss what implications can be gained from our study which we believe to be useful for designing effective teaching interventions for improving students' spatial skills and knowledge. Finally, we suggest some ideas for such teaching interventions. 


\section{Theoretical framework}

\section{Spatial reasoning skills in 3D geometry problem solving with 2D representations}

Pittalis and Christou (2010) identified four types of reasoning with 3D geometry: the representation of 3D objects, spatial structuring, conceptualisation of mathematical properties and measurement. These four types of reasoning are closely related to more general spatial skills such as spatial visualisation, orientation (e.g. remaining unconfused by changing orientations) or relations (e.g. mentally rotating objects) (p. 195). Lowrie et al. (2018) also list mental rotation, spatial orientation and spatial visualisation as spatial reasoning skills. In this paper, we particularly focus on the skills related to manipulating internal/external representations of 3D shapes and reasoning with geometric properties, because geometrical reasoning is characterised by the interaction between these two aspects, the visual (figural) and the conceptual (Duval 2017, p. 63).

Fischbein (1993) proposed the notion of "figural concept" such that, while a geometrical figure (such as a square) can be described as having intrinsic conceptual properties (in that it is controlled by geometrical theory), a geometrical figure is not solely a concept but rather an image too (ibid, p. 141). Various representations of geometrical shapes, such as diagrams, are used in geometry problems so that problem solving often involves encoding information from these representations (Lowrie 2012, p. 151). In a study of students working with 3D geometric shapes, Pittalis and Christou (2013, p. 676) identified the skills of coding information, e.g. manipulating and constructing nets, constructing 2D drawings of 3D shapes, and translating from one representation mode to another and decoding information, e.g. interpreting structural elements of representations of 3D shapes and geometrical properties. Battista et al. (2018, p. 195) also propose coding/decoding skills, taking spatial visualisation as "mentally creating and manipulating images of objects in space, from fixed or changing perspectives on the objects, so that one can reason about the objects and actions on them, both when the objects are and are not visible" and refer to property-based spatial analytic reasoning ("decomposes objects into their parts using geometric properties to specify how the parts or shapes are related, and, using these relationships, operates on the parts").

Based on the above, in this paper we take the following two skills as core spatial reasoning skills for problem solving with $3 \mathrm{D}$ geometrical shapes represented in 2D:

- Spatial visualisation (coding): mental manipulations of visual images of shapes including rotating, transforming the given diagrams to another form, reorienting, drawing nets and adding additional lines.

- Property-based spatial analytic reasoning (decoding): interpreting the structural elements of shapes and decomposing objects into their parts using geometric properties for reasoning and decision-making

\section{Use of spatial reasoning skills and domain-specific knowledge}

In geometry, it is known that certain images of geometrical shapes can disturb students' geometrical reasoning. A well-known example is the effect of prototypical images such 
as 'slanted' images for parallelograms in that through such images students do not include rectangles or squares as special cases of parallelograms even if they can state an appropriate definition of a parallelogram (e.g. Fujita 2012). Aspinwall et al. (1997) conceptualized the limitation of the use of visual imaginary as an uncontrollable image, described as "preventing the opening up of more fruitful avenues of thought, a difficulty which is particularly acute if the image is vivid" (p. 301). Another example is the dual process in which errors are "the result of either an uncontrolled intuitive thought process (no conflict-detection) or an unsuccessful intervention of the analytical processing system" (Van Hoof et al. 2013, p. 155, see also Gillard et al. 2009).

Llinares and Clemente (2019) refer to configural reasoning, which coordinates visual/figural and conceptual aspects of geometrical shapes. In this paper, we consider that students' statement around elements, properties and concepts of geometrical figures, i.e. domain-specific knowledge (Chinnappan et al. 2012) might also be important for coordinating visual and conceptual aspects in addition to general spatial reasoning skills described in the previous section. For example, Ufer et al. (2008) studied procedural knowledge (e.g. simple calculations in geometry) and declarative knowledge (e.g. recalling basic definitions, theorems, and concepts) and problem solving skills. They identified declarative knowledge to be the strongest influence on successful proving in geometry, with such knowledge being in the form "A is B", "in X, A is B", etc., in students' discursive expression. In this paper, we take students' statement around elements, properties and concepts of geometrical figures as "domain-specific knowledge" of geometrical shapes. Their property-based spatial analytic reasoning is represented by the use of knowledge, often accompanying by verbal or written explanations that use the word "because". Whether correct knowledge or not, in principle domainspecific knowledge in geometry (i.e. the concepts, principles and conventions unique to that domain and represented by in X, A is B, such as "In a cube, all faces are squares") plays a kind of coordinating role between spatial visualisation and property-based spatial analytic reasoning (of course, if students have incorrect knowledge about shapes then this can lead to incorrect answers).

\section{Types of answers in 3D geometry problem solving}

In 3D geometry, the use of spatial reasoning skills and domain-specific knowledge can lead both to successful and unsuccessful problem solving. In our previous studies (Fujita et al. 2017), by using data from 455 grades 7-9 students, geometric reasoning with 3D shapes were classified into five types in terms of mental manipulations of figures and reasoning based on geometric properties. In this paper, we also use the categories to see students' use of spatial reasoning skills and domain-specific knowledge in more integrated ways, described as follows:

- Type 1: Incorrect answer led by two-dimensional or intuitive global judgments without any explicit use of spatial visualisation and property-based spatial analytic reasoning;

- Type 2A: Incorrect answer only led by property-based spatial analytic reasoning but with logical gaps or influenced by visual information and/or incorrect/inappropriate domain-specific knowledge; 
- Type 2B: Incorrect answer with only spatial visualisations but influenced by visual information and/or incorrect/inappropriate domain-specific knowledge;

- Type 2C: Incorrect answer with the use of spatial visualisation and property-based spatial analytic reasoning but influenced by visual information and/or incorrect/ inappropriate domain-specific knowledge;

- Type 3: correct answer with valid use of spatial visualisation and/or property-based spatial analytic reasoning with correct domain-specific knowledge

This classification enables us to identify what makes students' problems solving successful/unsuccessful in their use of spatial reasoning skills and domain-specific knowledge, represented by various forms (e.g. verbal, written) of students' answers. For example, suppose a student answered "AB=CD" for Q2-(2) (Fig. 1). Then, this student answered $\mathrm{AB}=\mathrm{CD}$ because of the use of incorrect knowledge "in a cube all sides and edges have the same length" (type 2B). If a student has knowledge that 'in a cube, diagonals of each face are equal', then they might be able to reason triangle $\mathrm{ABC}$ in Fig. 1 question Q3-(2) is equilateral, despite it does not look like an equilateral triangle in the given diagram (type 3). Another student might reason " $\mathrm{CA}=\mathrm{CB}$ because they are diagonal" and conclude it is an isosceles triangle but no further manipulations are used (type 2A), mentally rotate the given diagram and then conclude isosceles, but not stating domain-specific knowledge explicitly to reason why (type $2 \mathrm{~B}$ ), or mentally extract triangles but no manipulations for the side $\mathrm{AB}$ and used " $\mathrm{CA}=\mathrm{CB}$ because they are diagonal" but conclude it is isosceles (type 2C). Also, knowledge about isosceles triangle is "inappropriate" in this problem context as its use is unlikely to lead to a correct answer.

\section{Methodology}

\section{The survey}

By selecting students in Japan, we were aware that the students would have had various learning experiences involving basic knowledge about 3D shapes including cubes, cuboids, prisms, pyramids, nets and how to represent 3D shapes in $2 \mathrm{D}$, by following the Japanese curriculum:

- In primary schools, cube and cuboid are introduced in G4, and then in G5, they further learn prisms, cylinder, nets and 2D representations of 3D shapes. Also by G4, they have learnt different triangles (equilateral, isosceles, scalene, right angled, etc.), quadrilaterals (square, rectangle, rhombus, parallelogram), parallel/ perpendicular lines, angles, diagonals of quadrilaterals, etc. Although deductive proofs are not studied, students are encouraged to explain their reasoning with practical activities in mathematics lessons;

- In secondary schools, G7, students study geometrical constructions, symmetry and selected properties of solid figures (names of 3D shapes, nets, surface areas and volume) informally, but logically, to establish the basis of the learning of proof which is introduced in G8 in a context of congruent triangles. 


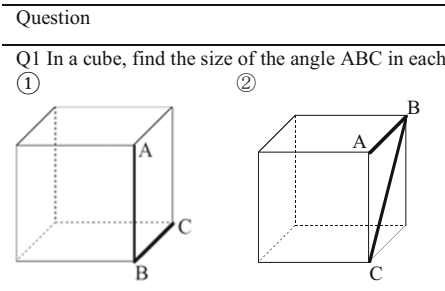

Q2 In a cube, which is longer, $\mathrm{AB}$ or $\mathrm{CD}$ ? Choose your answers from a)-d) for each case. Also write your reasons why.

(1)
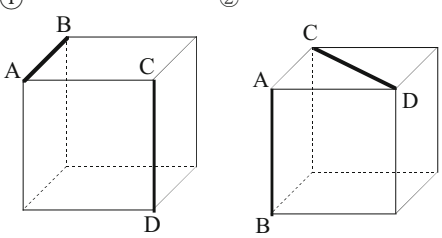

a) $\mathrm{AB}$ is longer

b) $\mathrm{CD}$ is longer

c) $\mathrm{AB}=\mathrm{CD}$

a) I am not sure which is longer.

Q3 In a cube, can you identify the shapes $A B C D$ and $\mathrm{ABC}$ ? Choose your answer from a)-e) for each case.

(1) A, B, C \& D are mid points of each edge

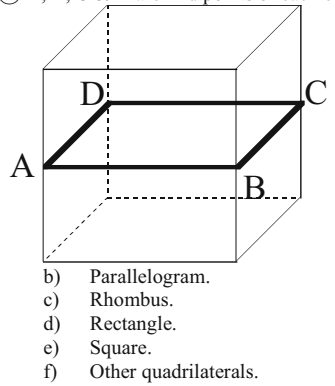

Q3 In a cube, can you identify the shapes $\mathrm{ABCD}$ and $\mathrm{ABC}$ ? Choose your answer from a)-e) for each case. (2)

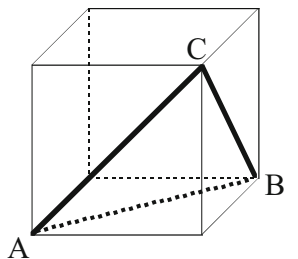
a) Right angled triangle.
b) Isosceles triangle.
c) Right angled isosceles triangle.
d) Equilateral triangle
e) Scalene triangle.

Spatial reasoning skills $\quad$ Domain-specific knowledge

SV: Recognise faces of cube,
extract angles, etc.

PSA: 90 or 45 based on the Faces are squares, all angles in square are equal or 90, diagonals of properties of squares

SV: Recognise faces of cube, transfer $A B$ and $C D$ to the same face, mentally create a square, move mentally $\mathrm{AB}$ to compare $\mathrm{CD}$ etc.

PSA: for Q2-(2), deducing $\mathrm{CD}>\mathrm{AB}$ because $C D$ is a diagonal of cube and diagonals of square are longer than the sides, etc.
SV: Recognise faces of cube, mentally dissect a cube with $\mathrm{ABCD}$, mentally superpose $\mathrm{ABCD}$ to one of the faces of cubes, move $\mathrm{ABCD}$ in parallel etc.

PSA: $\mathrm{AD}$ and $\mathrm{BC}, \mathrm{CD}$ and $\mathrm{BA}$ are parallel, and all angles are equal, and therefore $\mathrm{ABCD}$ is a square.
$\mathrm{SV}$ : Recognise $\mathrm{ABC}$ as a triangle, extract $\mathrm{ABC}$ mentally from cube, mentally compare $\mathrm{AB}, \mathrm{BC}$ and $\mathrm{CA}$, extract angle $\mathrm{ABC}$ etc.

PSA: $\mathrm{AB}, \mathrm{BC}$ and $\mathrm{CA}$ are diagonals of each face, and $\mathrm{AB}=\mathrm{BC}=\mathrm{CA}$. Therefore, it is equilateral triangle.
$\mathrm{AB}$ is an edge of a cube but $\mathrm{CD}$ is a diagonal of cube, diagonals of square are longer than the sides, etc.
Mid points bisect lines, in cube, there are parallel edges and faces, etc.

In cube diagonals of each face are equal.

SV: spatial visualisation

PSA: property-based spatial analytic reasoning

Fig. 1 Question items for primary and lower secondary schools

In G9, students learn similar figures and properties of circles, drawing on their consolidated capacity to use proof in geometry and Pythagorean Theorem with both $2 \mathrm{D}$ and $3 \mathrm{D}$ shapes. 
In order to answer RQ1, a survey was conducted with 261 grade (G)4, 213 G5, 209 G6, 225 G7, 224 G8 and 225 G9 students (in total 1357 grades 4-9 students aged from 10 to 15 years old) from state Japanese primary and lower secondary schools. The survey items do not appear in Japanese textbooks and we consider that, in using these, we would be able to identify "geometric thinking beyond that which is typically elicited in conventional lessons" (Fujita et al. 2017, p. 100). Q1-3 were answered by all the students. For each item, oblique parallel projections of cubes (as in Figs. 1 and 2) were used as such representations would be familiar to students (Kondo et al. 2014; see, also, Parzysz 1991). They were designed using cubes, because (a) it is a familiar shape for students and also (b) it is possible to include mathematically challenging problems which can help reveal students' use of spatial reasoning skills, and domain-specific knowledge. Expected use of spatial reasoning skills, and domain-specific knowledge, through our a priori analysis, are summarised in Figs. 1 and 2 (note for some question, one of spatial reasoning skills might be enough to answer a question correctly, e.g. Q3-(1), Q4 etc.). Also, for each question, duplicating knowledge is omitted; e.g. for Q5 "In a cube, the diagonals of each face are equal" might be used). Based on our previous studies and theoretical framework, students across G4-6 might find Q2-(2) or Q3-(2) to be difficult as they need to control their spatial reasoning skills by using domain-specific knowledge, and similarly across G7-9 for Q5, resulting more type $2 \mathrm{~A}-2 \mathrm{C}$ answers to these questions.

While the survey provides an overall account of the students' use of spatial reasoning skills and knowledge with $3 \mathrm{D}$ shapes (characterised through the survey questions with cubes), it has certain limitations. In particular, from the survey, we could only judge the types of the answers from "written" responses, which might influence students' thinking processes. Also, some students might not be familiar with writing their reasoning in a test situation. We are aware of these limitations in

\begin{tabular}{lll}
\hline Question & Spatial reasoning skills & Domain-specific knowledge \\
\hline $\begin{array}{l}\text { Q4 R, P and Q are the mid points of } \mathrm{AB}, \mathrm{EF}, \text { and } \mathrm{DC} \text { in a } \\
\text { cube. What is the size of the angle RPQ? }\end{array}$ & $\begin{array}{l}\mathrm{SV}: \mathrm{Recognise} \mathrm{RPQ} \text { as an angle, } \\
\text { move RPQ in parallel, etc. }\end{array}$ \\
& $\begin{array}{l}\text { PSA: AD//PR and } \mathrm{AE} / \mathrm{PQ} \text {, so the } \\
\text { angle } \mathrm{DAE} \text { and } \mathrm{RPQ} \text { are equal, and } \\
\text { therefore } 90 .\end{array}$
\end{tabular}

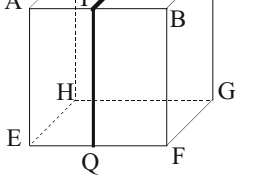

Q5 What is the size of the angle DEB in a cube? Write your reasons why.

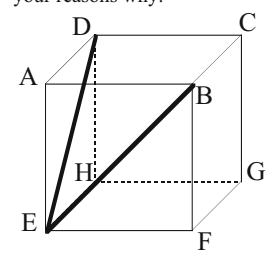

SV: Recognise DEB as a triangle, In equilateral triangle, all angles are extract DEB mentally from cube, mentally compare DE, EB and $\mathrm{BD}$, extract angle $\mathrm{ABC}$ etc.

PSA: DE, EB and BD are diagonals of each face, and $\mathrm{DE}=\mathrm{EB}=\mathrm{BD}$. Therefore, it is equilateral triangle and the angle is 60 . equal.

Fig. 2 Additional question items for lower secondary schools 
our study, but we believe we managed to extract useful information on students' use of spatial reasoning skills and domain-specific knowledge from the survey, as shown in our results section.

\section{Lesson observation}

In order to gain further information which might have influenced our survey data, in particular students' thinking processes, we also conducted a classroom-based study in which G6 students undertook Q5. We are aware that Q5 was probably too difficult for such students but, as an exploratory stage in lesson implementation, we wanted to see how the G6 students would tackle Q5.

A 45-min lesson was implemented in February 2019 by Mr. T with more than 20 years of teaching experience. Also the lesson was planned with the authors. The 34 students undertook the pre-/post-survey (using the tasks shown in Figs. 1 and 2) in December 2018 and March 2019 respectively. The first four authors observed the lesson and undertook their notes individually, video-recoded some of individual/group work and then these notes were shared in the post-lesson discussion and analysis. We focus on a student (SW) and examine her learning processes by describing the use of spatial reasoning skills and domain-specific knowledge in terms of the types of answers during the lesson. Student SW was randomly chosen by two of the observers at the beginning of the lesson ad hoc, and we recorded her thinking processes in lesson observation notes and video clips (a standard method of lesson observation data collection in Japan).

\section{Data analysis}

We first identified groups of geometry problems with similar difficulty levels. We created a $1357 \times 6$ matrix for both primary and secondary schools and a $674 \times 8$ matrix for secondary schools' results with two variables (not correct, correct), and conducted an analysis using the Item Response theory approach (Sijtsma and Molenaar 2002) with R 3.3.3 with the packages "ltm" and "irtoys". We undertook 2-parameter logistic (2PL) model analysis with the following two parameters: difficulties and discriminations. We use the 2PL model because the model is appropriate to see overall relationships of items in terms of the difficulties of each item (the difficulties parameter), and differences between students at different grade levels (the discrimination parameter).

Once we gained an overall picture of the students' responses, we then examine the students' written explanations in the survey further as such explanations represent evidence of what spatial reasoning skills and domain-specific knowledge which students were using to try and solve the geometric problems (this addresses RQ1). For the primary school students' responses, we paid particular attention to Q2 (for which G4-9 students were asked to write their explanation) and Q5 (for which G7-9 were asked to write their explanation). In analysing the written responses to Q2-(2) and Q5, we use the types of answers to account for students' use of spatial reasoning skills and domain-specific knowledge as well as in what ways some students had difficulties reaching correct answers for the given problems. Informed by our a priori analysis shown in Figs. 1 and 2, we first coded 
selected sampled examples individually, compared our preliminary coding, and continued our discussion until we agreed. We also did lesson observations together, and have had more than $5 \mathrm{~h}$ of face-to-face discussions to agree how to code examples from the lessons. After this, we divided data into three sets and three of the authors completed the coding for Q2-(2) and Q5. The examples from student SW were coded by two authors independently, and no major disagreements were found. Then the analysis was shared with the other authors and agreed. Finally, we discuss our findings and, by integrating our findings from the survey and lesson observation, we consider implications for designing future interventions (thereby addressing RQ2). In this collaborative way, we are fairly confident of reliability of data analysis and coding.

\section{Findings from the survey}

\section{Overall performance}

Analyses of the survey data for Q1-3 and Q4\&5 are summarised in Tables 1 and 2 (\%s for correct answers).

From Table 1, showing the percentage correct for Q1-3, it can be seen that almost all students from G4 knew that the angle between adjacent edges of a cube is 90 degrees (Q1-(1)), that the angle between an edge and the diagonal of a face is 45 degrees (Q1-(2)), and that all the edges of a cube are congruent (Q2-(1)). In general, primary students found Q2-(2), Q3-(1), and Q3-(2) to be more difficult than the first three questions. For the secondary schools, almost all students from $\mathrm{G} 7$ managed to answer correctly Q1-(1), Q1-(2), Q2-(1) and Q4. For Q2-(2), there is a higher proportion of correct responses in G9, but about $30 \%$ of G7 and 8 were not able to answer this question correctly. About $40 \%$ of G7 students answered Q3-(1) incorrectly. About 20\% of G4, 30\% of G5 and 20\% of G5-8 students selected "rectangle" as their answers, but more than $80 \%$ of all the grades selected either "rectangle" or "squares". This indicate almost all students can recognise angles such as DAB as 90 degrees, but those who answered "rectangle" might have influenced by "how it looks". All students found Q3-(2) and Q5 to be fairly difficult to answer.

Tables 3 and 4 summarise details for Q2-(2) and Q3-(2).

Table 3, showing the details for Q2-(2), suggests that in primary schools (G4-6) about $30 \%$ of students considered that the side of the cube (AB) is the same length as

Table 1 Percentage correct for Q1-3

\begin{tabular}{lllllll}
\hline & $1-(1)$ & $1-(2)$ & $2-(1)$ & $2-(2)$ & $3-(1)$ & $3-(2)$ \\
\hline G4 $(N=261)$ & 94.3 & 74.7 & 91.6 & 47.5 & 63.2 & 12.6 \\
G5 $(N=213)$ & 95.8 & 86.9 & 87.8 & 51.2 & 49.8 & 16.9 \\
G6 $(N=209)$ & 94.3 & 85.2 & 95.2 & 59.3 & 68.4 & 36.8 \\
G7 $(N=225)$ & 97.3 & 89.3 & 94.2 & 67.1 & 61.8 & 34.2 \\
G8 $(N=224)$ & 96.4 & 90.6 & 95.5 & 70.1 & 66.5 & 35.3 \\
G9 $(N=225)$ & 99.6 & 96.0 & 95.1 & 84.4 & 78.2 & 52.4 \\
\hline
\end{tabular}


Table 2 Percentage correct for Q4\&5

\begin{tabular}{llr}
\hline & Q4 & Q5 \\
\hline G7 $(N=225)$ & 92.9 & 8.4 \\
G8 $(N=224)$ & 91.5 & 13.8 \\
G9 $(N=225)$ & 95.1 & 37.8 \\
\hline
\end{tabular}

the diagonal of a face (CD); see Q2-(2). By G8 about $18 \%$ of students still answered $\mathrm{AB}=\mathrm{CD}$ in the given diagram.

Table 4, showing the details for Q3-(2), suggests that, up to G8, more than $30 \%$ of students answered that the triangle was an isosceles but not equilateral, while more than $20 \%$ of students in G4-8 considered the triangle in the given diagram contained a right angle. Table 4 also shows that the majority of students answered the triangle would be an isosceles, or right-angled isosceles, indicating that they might have been influenced by the visual appearance of the given diagram. Q3-(2) requires more than one step of reasoning (identifying diagonals in each face $\rightarrow$ diagonals are equal $\rightarrow$ equilateral triangle), and it is expected this problem might be difficult for G6, but it was rather surprising there is no improvement until G9.

The above data suggest that, overall, there might be gaps across the grades between the set of questions (Q1-(1), Q1-(2), Q2-(1)), (Q2-(2), Q3-(1)) and question (Q3-(2)) (and [Q5] in secondary schools). In order to confirm this, we conducted 2LPM analysis in order to gain further insight into the findings, in particular examining each question item in terms of their difficulties. Figure 3 (G4-9, Q1-(1) Q3-(2)) and Fig. 4 (G7-9, Q1-(1) Q5) show the relationships between each question in terms of the difficulties, and discriminations, alongside the best-fitted logistic curves for each question.

The relative difficulties listed in Figs. 3 and 4 indicate the following for G4-9 students: the relative difficulties for Q1-(1), Q1-(2) and Q2-(1) are similar; the difficulties of Q2-(2) and Q3-(1) are similar and more difficult than the first 3 questions, and Q3-(2) is confirmed as more challenging than the other questions until G9. Q5 is the hardest question for secondary school students. These two figures and 2PLM suggest that there are gaps between (Q1-(1), Q1-(2), Q2-(1)), (Q2-(2), Q3-(1)) and (Q3-(2)) (and [Q5] in secondary schools). The values of the discrimination for each questions are above 1 , indicating all the items discriminate relatively well between low-skilled

Table 3 Details of answers for Q2-(2), percentages

\begin{tabular}{llllll}
\hline Q2-(2) & AB is longer & CD is longer & AB=CD & I do not know & No answer \\
\hline G4 $(N=261)$ & 11.1 & 47.5 & 33.3 & 2.7 & 5.4 \\
G5 $(N=213)$ & 12.2 & 51.2 & 31.0 & 4.7 & 0.9 \\
G6 $(N=209)$ & 7.7 & 59.3 & 30.6 & 1.9 & 0.5 \\
G7 $(N=225)$ & 9.8 & 67.1 & 21.3 & 1.8 & 0.0 \\
G8 $(N=224)$ & 9.4 & 70.1 & 17.9 & 2.2 & 0.4 \\
G9 $(N=225)$ & 9.3 & 84.4 & 4.4 & 1.8 & 0.0 \\
\hline
\end{tabular}


Table 4 Details of answers for Q3-(2), percentages

\begin{tabular}{lcccccc}
\hline Q3-(2) & Right angled & Isosceles & Right-angled isosceles & Equilateral & Scalene & No answer \\
\hline G4 $(N=261)$ & 11.9 & 46.7 & 10.0 & 12.6 & 16.5 & 2.3 \\
G5 $(N=213)$ & 16.4 & 42.3 & 9.9 & 16.9 & 14.1 & 0.5 \\
G6 $(N=209)$ & 7.7 & 31.6 & 12.0 & 36.8 & 11.5 & 0.5 \\
G7 $(N=225)$ & 8.9 & 33.3 & 11.6 & 34.2 & 12.0 & 0.0 \\
G8 $(N=224)$ & 5.4 & 33.0 & 21.4 & 35.3 & 4.0 & 0.9 \\
G9 $(N=225)$ & 4.9 & 26.7 & 12.0 & 52.4 & 4.0 & 0.0 \\
\hline
\end{tabular}

and high-skilled students. The overall analysis also suggests that there are difficulty gaps in Q2-(2), Q3-(2) or Q5, and we further explore what spatial reasoning and domain-specific knowledge were used to answer these questions correctly/incorrectly by focusing on Q2-(2) and Q5.

\section{Reasoning skills and knowledge used by G4-6 students for Q2-(2)}

When we examined Q2-(2) (in which G4-6 students were asked to write their reasoning), we noted that students whose explanations included the word "a diagonal (of square)" or "slanting" tend to answer this question correctly, although not many students provided such explanations. For example, 18/216 G4, 38/213 G5, and 57/209 of G6 students used a word "a diagonal of square" in their reasoning why $\mathrm{CD}>\mathrm{AB}$, and of these students who used "a diagonal", $61.1 \%, 89.5 \%$ and $75.4 \%$ of G4-6 students answered Q2-(2) correctly. This indicates that these students used their domain-specific knowledge that "in a square, diagonals are longer than sides", a type 3 answer (some of them might have also used spatial visualisation skills but we could not see explicitly from the written answers). However, if students have "incorrect" domain-specific knowledge for cubes such as "in a cube, all the sides including diagonals are equal (in Japan, 'sides (辺)' for both 2D and 3D shapes but not 'edge'), then such students' property-based spatial analytic reasoning was represented by ' $\mathrm{AB}=\mathrm{CD}$ ' as ' $\mathrm{AB}$ and $\mathrm{CD}$ are 'sides' of cube, and in cube all sides are equal". The

\begin{tabular}{lll}
\hline Question & Difficulty & Discrimination \\
\hline Q1-(1) & -2.1719 & 2.5312 \\
Q1-(2) & -1.4689 & 2.0598 \\
Q2-(1) & -1.9535 & 2.0939 \\
Q2-(2) & -0.5624 & 1.2593 \\
Q3-(1) & -0.5777 & 1.4817 \\
Q3-(2) & 0.7593 & 1.4569
\end{tabular}

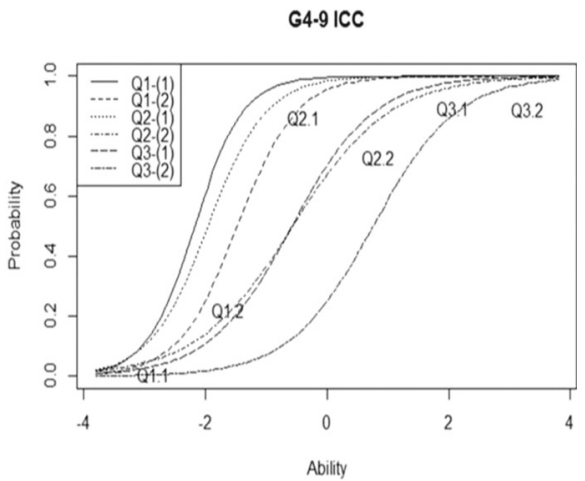

Fig. 3 Values in each question (left) \& Item characteristic curves (right) for G7-9 


\begin{tabular}{lll}
\hline Question & Difficulty & Discrimination \\
\hline Q1-(1) & -2.2892 & 3.1142 \\
Q1-(2) & -1.7758 & 2.2457 \\
Q2-(1) & -1.9978 & 2.4942 \\
Q2-(2) & -1.0354 & 1.3378 \\
Q3-(1) & -0.6812 & 1.7930 \\
Q3-(2) & 0.3422 & 1.5623 \\
Q4 & -2.224 & 1.5356 \\
Q5 & 1.0455 & 1.9622
\end{tabular}

G7.9 ICC

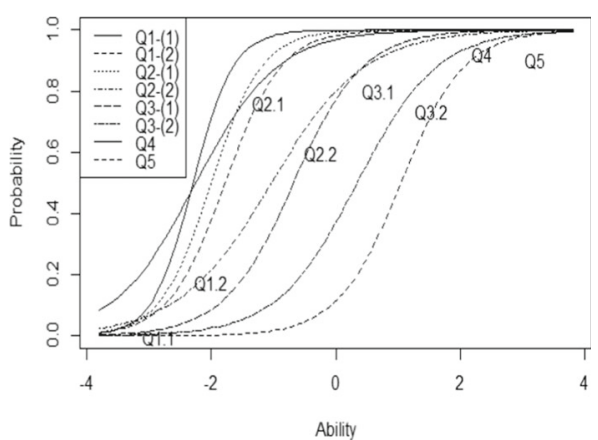

Fig. 4 Values in each question (left) \& Item characteristic curves (right) for G7-9

majority of the students who answered $\mathrm{AB}=\mathrm{CD}$ gave this explanation, i.e. type $2 \mathrm{~A}$ answer. This might indicate that the use of correct domain-specific knowledge (in this case "faces of cube are squares" and "in a square, diagonals are longer than sides") explicitly is important to solve this problem.

We also noted that some students additionally explicitly used spatial visualisation skills as evidenced by students' answers such as 'It is easier to solve this question by imagining a net of a cube" (G6) or by them adding further drawings, e.g. "when we compare a side of square and other lines, then the line becomes more distant when it goes middle", as illustrated in Fig. 5 Although not many in our sample, such answers were categorised as type 3 .

\section{Reasoning skills and knowledge used by G7-9 students for Q5}

Table 5 summarises students' answer for Q5 in terms of the types of errors:

- Type 1: Incorrect answer led by 2-dimensional or intuitive global judgments, e.g. answers such as $90^{\circ}$ with no reason stated, "looks like $90^{\circ}$ ", "Maybe $\left(90^{\circ}\right)$ " without stating any reasons or manipulations in their answers.

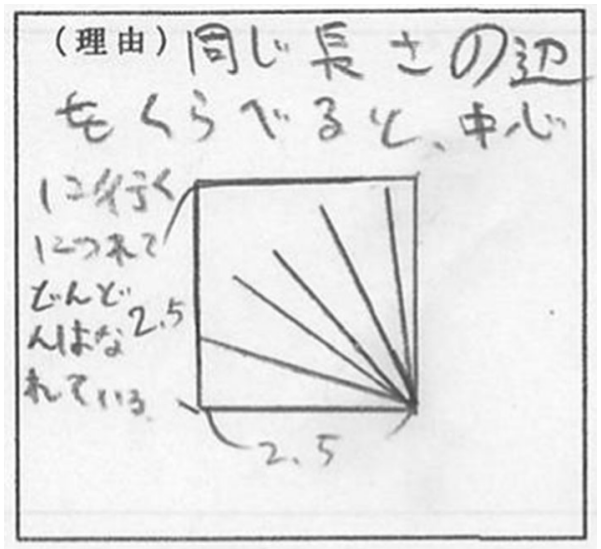

Fig. 5 An explanation for Q2-2 
Table 5 Types of answers for Q5, percentages

\begin{tabular}{lllllrl}
\hline Type & 1 & 2A & 2B & 2 C & 3 & No answer \\
\hline G7 $(N=225)$ & 20.0 & 41.3 & 12.0 & 5.3 & 8.4 & 12.9 \\
G8 $(N=224)$ & 21.4 & 35.3 & 11.6 & 2.7 & 13.8 & 15.2 \\
G9 $(N=225)$ & 18.7 & 17.8 & 13.3 & 3.1 & 37.8 & 9.3 \\
\hline
\end{tabular}

- Type 2A: Incorrect answer only led by property-based spatial analytic reasoning but with logical gaps or influenced by visual information, and/or incorrect/inappropriate domain-specific knowledge, e.g. answers such as " $\angle \mathrm{AEF}=90^{\circ} .90 / 2=45^{\circ}$ ", "the face $\mathrm{ABFE}$ and $\mathrm{ADHE}$ are perpendicular to each other, and BE and $\mathrm{ED}$ are on these faces, so $90^{\circ}$ ", " $\angle \mathrm{BEA}+\angle \mathrm{DEA}=45^{\circ}+45^{\circ}=90^{\circ}$ ", etc.

- Type 2B: Incorrect answer with only spatial visualisations but influenced by visual information and/or incorrect/inappropriate domain-specific knowledge, e.g. answers such as "(drawing a net), and then $45^{\circ}+45^{\circ}=90^{\circ}$ ", "(changing the orientation of the given figure) $90^{\circ}$ ", "Looking the given from the above (mentally), it will be $90^{\circ}$ ", etc.

- Type 2C: Incorrect answer with the use of spatial visualisation and property-based spatial analytic reasoning but influenced by visual information and/or incorrect/ inappropriate domain-specific knowledge. Here, triangle $\mathrm{BDE}$ is (mentally or on the diagram) extracted and seen as isosceles, and then inappropriate knowledge is used, e.g. "in $\triangle \mathrm{BDE}$, because $\angle \mathrm{D}=\angle \mathrm{E}=45^{\circ}$ and it will be $90^{\circ}$ ", " $\angle \mathrm{DAB}$ is $90^{\circ}$ and $\angle \mathrm{BED}$ is just an adjusted from $\angle \mathrm{DAB}$, so it is $90^{\circ}$ ", "in a triangle $\mathrm{BDE}, \angle \mathrm{B}=90^{\circ}, \angle \mathrm{D}=60^{\circ}$ and therefore $30^{\circ}$ ".

- Type 3: correct answer with valid use of spatial visualisation and/or property-based spatial analytic reasoning with correct domain-specific knowledge, e.g. answers such as "in triangle $\mathrm{BDE}, \mathrm{DE}=\mathrm{EB}=\mathrm{BD}$ and therefore $\angle \mathrm{BED}=60^{\circ}$ ", "EB, $\mathrm{ED}$ and $\mathrm{BD}$ are all the diagonal of the same square. Since all the sides are equal to each other, it is an equilateral triangle (and therefore $60^{\circ}$ )".

From Table 5, giving the percentages of types of answers for Q5, students in G7 and G8 did use various approaches to solve Q5, but they relied either on only propertybased spatial analytic reasoning (type 2A) or only spatial visualisation (type 2B) as well as being influenced by the perceptual appearance of the given diagram. More G9 students answered Q5 correctly (37.8\%) compared to G7 and 8, but 18.7\% of them still relied on global judgement or provided no explanation for their answer.

When we examined the students' explanations for their answers, it seems that students who used "in a cube, diagonals of each face are equal" were likely to give a correct answer. In G9, for example, 89 (37.8\% of G9) students answered this question correctly, and within these 89,50 of them explicitly used such knowledge to deduce the triangle is an equilateral. For G8, 32 students answered Q5 correctly, and 15 of them used "diagonals" in their explanations (in G7 but 6 of 26 who answered Q5 correctly did so). This might imply, similar to the case for Q2-(2), that domain-specific knowledge coordinates between spatial visualisation and property-based spatial analytic reasoning. 


\section{Summary of the survey results}

Reflecting on the survey results and analyses described above, the following matters are suggested in terms of the use of spatial reasoning skills and domain-specific knowledge and types of answers:

- Descriptive statistics and 2PLM analysis that we present suggest that gaps (in the context of our survey questions) exist between (Q1-(1), Q1-(2), Q2-(1)), (Q2-(2), Q3-(1)) and (Q3-(2)) (and [Q5] in secondary schools) across the grades.

- Examining the students' explanations for Q2-(2) and Q5 further in terms of the types of answers, one of the spatial reasoning skills (such as Type 2A or 2B), or two skills without domain-specific knowledge (Type 2C), might not be enough to answer this question correctly. In other words, to support students' successful problem solving from type $1 / 2 \mathrm{~A} / 2 \mathrm{~B} / 2 \mathrm{C}$ to 3 , it is important to encourage them not only to use spatial reasoning skills but also to make it explicit what knowledge can be used.

These findings imply (a) more research is needed to understand further the processes when students use spatial reasoning skills and domain-specific knowledge; in particular, considering how to support students successful with types $1 / 2 \mathrm{~A} / 2 \mathrm{~B} / 2 \mathrm{C}$ to develop to be successful with type 3 , and (b) learning opportunities could be provided much earlier than G8 as data from Q3-(2) point to 3 years of no improvement. This is important as in G9 students learn the Pythagorean theorem and apply this to find the lengths of diagonals in a cube, but about $30 \%$ of the students might not be able to answer Q2-(2) and 65\% unable to answer 3-(2) at the end of G8 respectively, which can lead G9 students to learn superficial procedures to find the length by using the theorem without seeing the structure of cubes, for example.

\section{Findings from lesson observation}

\section{Overall lesson progressions}

As stated in the methodology, 34 students worked with Q5 during a 45-min lesson. In the lesson, Mr. T first introduced the problem (Q5), confirming that the objective of the lesson was to find the size of angle BED. The first stage of the lesson was for the students to attempt to solve the question individually. As expected, at this stage, they
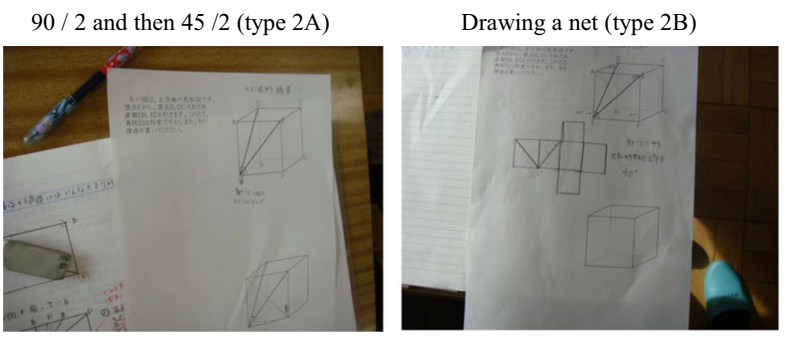

Changing orientations (type 2B)

Fig. 6 Students' initial attempts 
used a range of different spatial reasoning skills but almost no student concluded the angle might be $60^{\circ}$. Many of them used only property-based spatial analytic reasoning (type 2A) or spatial visualisation skills (type 2B) (Fig. 6). At this stage, it is not clear what domain-specific knowledge was used for many students.

The next stage of the lesson was for the students to start exchanging their ideas and answers, but the diversity of answers and approaches they had used meant that they could not improve their answers or extend their initial ideas through discussions.

SW, our case students, first visualised a pyramid from the given representation, and then rotated it to another pyramid (spatial visualisation, Fig. 7 left). After this, she seemed to visualise BDE as "an isosceles triangle" on the given diagram (Fig. 7 centre), and then tried to determine the size of the angle EDA, which she thought was $45^{\circ}$ (indicating type $2 \mathrm{C}$ answer). She then had a quick conversation with student $\mathrm{AO}$ who sat in front of her, whose answer was $90^{\circ}$. SW was not convinced by this as the angle "did not look like" $90^{\circ}$. During their conversation, SW and AO extracted a triangle (spatial visualisation), and, interestingly, almost identified the triangle would be an equilateral in order to deduce the size of the angle (property-based spatial analytic reasoning) as follows:

SW: (pointing the two lines the diagram) They are the same (lengths)...

AO: An isosceles triangle?

SW: An equilateral triangle?

AO Then, the sum of the angles is $180^{\circ}$. Then because all the angles are equal in equilateral, so 60, 60, 60, and this area (incorrectly used 'area' to say an angle), we can see this from the front (mentally rotating the given diagram) ... (then looking again the extracted triangle, and showed a confused face without any words).

However, SW was not convinced as the triangle on the diagram still did not "look like" an equilateral, but visualised the extracted triangle as an isosceles (Fig. 7 centre). At this point, both of them also did not use explicit domain-specific knowledge specifically related to cube (e.g. diagonal), but those related to triangles which is inappropriate to reach a correct answer (indicating Type 2C answer). SW remained unsure about her answer, and exchanged various opinions with other students who sat around her, including by holding paper (Fig. 7, right). Then AO started explaining why $90^{\circ}$, which is her answer, can look "narrower" in the given diagram:
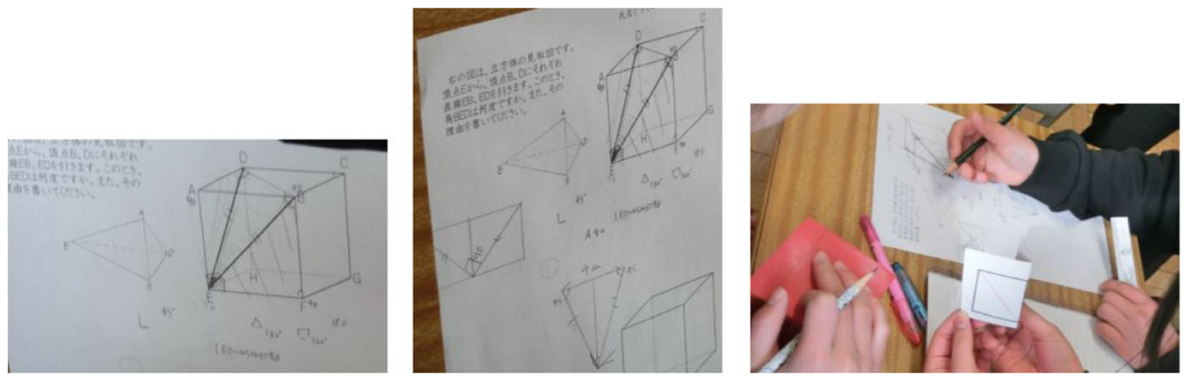

Fig. 7 SW's attempts 
AO: If you see my pencil from the above, it looks longer and from the side, it looks shorter. It is like that.

SW: Ah, I see now... I see, I need to open it (meaning to write a net, drawing additional diagrams, Fig. 6 right). So, it is $90^{\circ}$ ?

At this point, the statement "If you see my pencil from the above, it looks longer and from the side, it looks shorter. It is like that." by AO spurred SW to draw additional diagrams (spatial visualisation) and she used the property of an isosceles triangle to deduce the angle would be $90^{\circ}$ (property-based spatial analytic reasoning). This can be seen as type $2 \mathrm{C}$ as her answer was influenced by visual information and inappropriate knowledge in this problem context.

SW then went to see student OKY who explained to her that his answer was $60^{\circ}$ because of the equilateral triangle (Fig. 7 left, OKY drew another triangle to visualise an equilateral triangle, type 3). SW seemed to be quite satisfied with OKY's answer and explanation.

SW then tried to explain to student AO what she heard from student OKY, but SW was confused as she could not reproduce (Fig. 8 right) what she saw in OKY's answer. Our observational notes recorded her statements which were related to spatial visualisation such as "we can draw from the front (meaning the other direction)", "How did he do? How?", "Something was turned to an equilateral", etc. (Fig. 8 right) She also did not use statements such as "In cube, diagonals of each face are ..." at all, but used indicative words such as "there" or "here" to point the elements of the shapes out. At this point, she wrote $45^{\circ}$ and $60^{\circ}$ on her sheet. The thinking processes observed from SW indicate that SW managed to use both/either spatial visualisation and/or propertybased spatial analytic reasoning but because of the lack of domain-specific knowledge, her reasoning remained up to $2 \mathrm{C}$, and could not advance to type 3 by herself.

Soon after, Mr. T asked the student to stop working individually/with groups, and invited a few students to explain their reasoning. Student CA showed his answer as follows:

CA: First, here, first it is written from here (D and B) to there, the angle is written there, but when we rotated it ...

Students: (whispering) (my answer is) $22.5 \ldots$

CA: ... then it (the angle BED) will be the same angle of this one (in the rotated triangle in red in the figure below). When we consider with the rotated shape, then when we connect diagonals, diagonals, and diagonals of a square, and this is an equilateral triangle. The sum of the angle is $180^{\circ}$, and since their angles and sides are equal, we divide $180^{\circ}$ by 3 , and then it will be $60^{\circ}$.
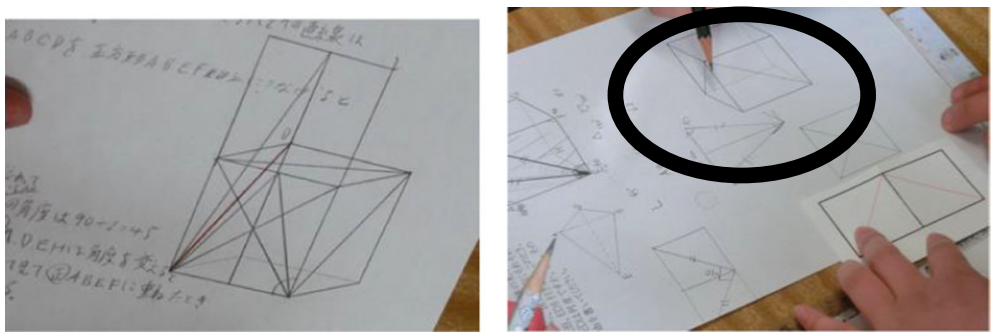

Fig. 8 OKT (left) and SW (right)'s answers 


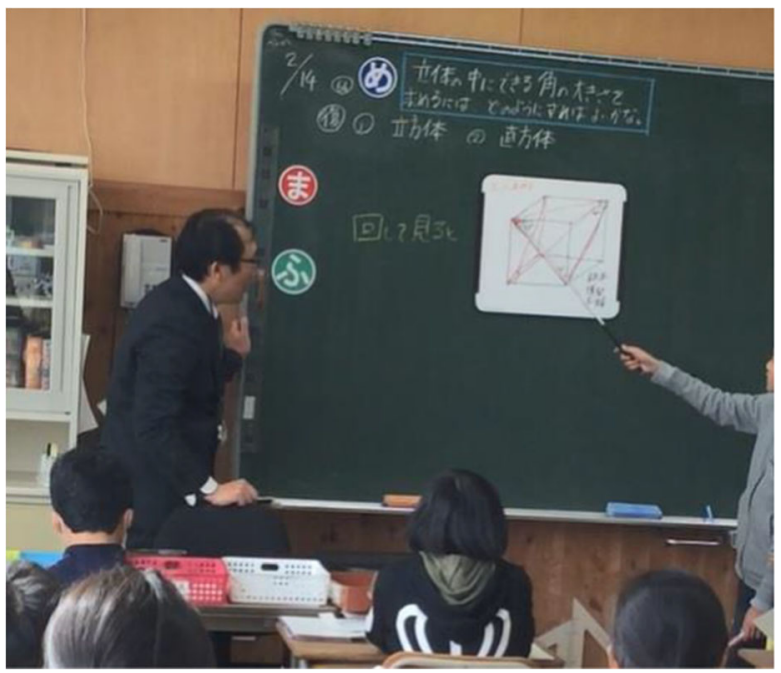

Fig. 9 CA's explanation why the angle BED is $60^{\circ}$

In his explanation in Fig. 9, CA rotated the given diagram (spatial visualisation, also on the board it is written “回して見ると (rotating and then see)”, indicating thinking by rotating, next to the diagram) and then used the diagonals of a square and the properties of triangles (sum of the angles and equilateral triangle) to deduce the angle is $60^{\circ}$ (property-based spatial analytic reasoning), type 3 answer. Here, domain-specific knowledge "in cube, diagonals of each face are equal" explicated and represented by "the diagonals of a square" coordinate his spatial visualisation and property-based spatial analytic reasoning and enabled him to identify the triangle is equilateral. This explanation (type 3) by CA seemed to be accepted by other students in the class, including SW who nodded during CA's explanation of his reasoning. After the explanation, SW managed to reproduce OKY's answer of $60^{\circ}$ (Fig. 10).

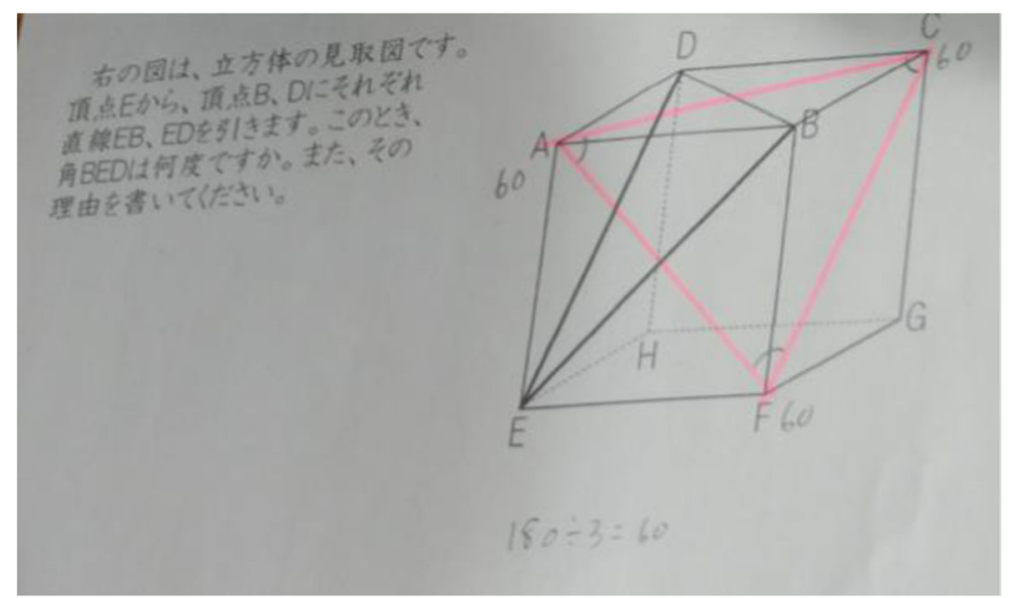

Fig. 10 SW's final answer 
Mr. T praised the student's efforts and CA's answer, asked them to finished their work, and complete the post-lesson problem (which, rather than finding angle BED, was to find the size of angle FCH). With that, the lesson finished.

\section{Pre-/post-survey results}

Prior to the research lesson in February 2019, students took the pre-survey (Fig. 1) in December 2018. One month after the lesson, the students took the post-survey (using the same survey). They were also asked to solve Q5 with the different angle (FAH). We do not claim that only one lesson make a positive impact on students' spatial reasoning skills, but we are interested in how the learning experience of the lesson made any changes in the students' answers. Table 6 summarises the results from the both surveys.

From Table 6, while the pre-survey results by this class was a bit lower than our main survey result, in the delayed post-survey their performance was generally an improvement on the pre-survey and the main survey. There was a significant difference in the pre-test $(M=3.89, \mathrm{SD}=1.64)$ compared to the post-test $(M=4.7, \mathrm{SD}=1.5), \mathrm{t}(33)=3.1, p<.005)$.

What is more, $41.2 \%$ of the students answered Q3-(2) correctly, which is higher than G8 performance (35.3\%) in the main survey. For the Q5 (finding FAH), 70.6\% of the students answered correctly. Considering that the post-survey was undertaken 1 month after the lesson, the results suggest that about 17 students (of 34) demonstrated good spatial reasoning skills as well as retaining what they learnt well after the lesson. Nevertheless, there were 6 students who did not show much difference from before to after the lesson.

Reflecting on the lesson observation, the pre/post-survey and its analyses described above, the following matters are suggested:

- G6 students used various spatial reasoning skills to tackle Q5. Similar to secondary school students, five types of answers were observed. It seems many students initially showed type $2 \mathrm{~A}$ and $2 \mathrm{~B}$ answers without extracting the triangle BDE.

- Discussions between the students were useful to exchange various ideas and answers, and this encouraged the students to think deeply about what they have done in terms of spatial visualisation and property-based spatial analytic reasoning, making them decide what triangle BDE would be. However, their answer remained as type $2 \mathrm{C}$. In order to harmonise the two spatial reasoning skills, the explicit use of domain-specific knowledge appropriate in the problem context might be more encouraged, exemplified by the case of student SW.

In the next section, we explore and integrate what our findings from the main survey and lesson observation imply in terms of our research question and the theoretical framework.

Table 6 Pre-/post-survey results

\begin{tabular}{llllllll}
\hline & $1-(1)$ & $1-(2)$ & $2-(1)$ & $2-(2)$ & $3-(1)$ & $3-(2)$ & Q5 (FAH) \\
\hline Pre-survey & 91.4 & 82.9 & 80.0 & 60.0 & 45.7 & 22.9 & - \\
Post-survey & 94 & 91.2 & 91.2 & 76.4 & 64.8 & 41.2 & 70.6 \\
G6 $(N=209)$ & 94.3 & 85.2 & 95.2 & 59.3 & 68.4 & 36.8 & - \\
\hline
\end{tabular}




\section{Discussion}

The importance of the spatial reasoning (e.g. Lowrie 2012; Lowrie et al. 2018; Mulligan et al. 2018; Lowrie et al. 2019) and relationships between different spatial skills and reasoning (Pittalis and Christou 2010, 2013) are documented in existing studies. The aim of this paper was to explore how the students across the grades use spatial reasoning skills and domain-specific knowledge to solve geometrical problems (RQ1), and what can be learnt to inform future interventions to improve students' spatial skills and reasoning (RQ2). By following Battista et al. (2018), we took spatial reasoning skills as spatial visualisation and property-based spatial analytic reasoning, and domain-specific knowledge which coordinates these two skills. We then categorised the types of answers and analysed the survey data from 1357 students across G4-9, and lesson observation from a G6 class.

Similar to existing studies, our survey results and lesson observation revealed that both spatial visualisation and property-based spatial analytic reasoning play important roles, but that, without appropriate domain-specific knowledge, students' reasoning can be influenced by the visual appearance of the geometrical objects in the problems (e.g. Ufer et al. 2008; Chinnappan et al. 2012). In particular, when problems have more than one step of reasoning (e.g. Q3-(2) or Q5 in our case), such problems remain challenging across the grades.

The contribution of our analysis of our data is that we found that the categorisation of five types of answers accounted for the ways students could not answer the survey problems correctly. For example, when students solved, for example, Q5, they might manage to use spatial visualisations such as rotating mentally the given diagrams, extract triangles, change their points of view etc. (type 2B for example), but they struggled to determine what the triangle would be. Similarly, they could use reasoning based on properties of shapes (property-based spatial analytic reasoning) but they still reached incorrect answers (e.g. type $2 \mathrm{~A}$ or type $2 \mathrm{C}$ ), because they were incorrect/ inappropriate. This might be caused by the given diagrams' visual appearance, i.e. the dual process (Van Hoof et al. 2013) or uncontrollable images (Aspinwall et al. 1997).

What is more, our findings suggest that the students who explicitly used domainspecific knowledge which was appropriate for the problem (e.g. "in cube, diagonals of each face are equal") were likely to reason correctly for the given problems (e.g. students' explanations for Q2-(2) or Q5 in the main survey or the case of CA). In particular, the effective use of domain-specific knowledge might enable students to break an impasse of reasoning (2A-2C) for successful reasoning (2C).

Given our findings discussed above, our answer to our RQ1 is that the students across the grades can use spatial reasoning skills, but for problems with more than one step of reasoning, students need to make their domain-specific knowledge explicit in problem solving, in particular which knowledge should be chosen and why. Fischbein (1993) calls this productive reasoning (also what Llinares and Clemente 2019, call configural reasoning), and we argue that it is domain-specific knowledge that coordinates visual and conceptual aspects in 3D geometry problem solving.

Our study reveals that although there are some improvement across the grades, certain problems such as Q3-(2) do not improve much from G6-8. Also many G9 students struggle to answer correctly Q5. This is an issue for, say, upper secondary schools in trigonometry, when students might be asked to solve various questions in 
trigonometry, e.g. finding areas of triangles formed in a cube, etc. However, existing studies also suggest that spatial reasoning skills can be improved by certain interventions (e.g. Lowrie et al. 2019). A positive result in the post-survey result (Table 6) suggests that it is possible to implement a lesson with a problem even G9 students find difficult, and even though it was only one lesson, about half of the G6 students retained what they experienced in this lesson well even after 1 month. This implies that current Japanese curriculum for 3D geometry should be re-examined, and more learning opportunities might be given to both primary and secondary school students in which they can not only exercise their spatial reasoning skills but also consolidate and share, discuss and explicitly use what they have as domain-specific knowledge for productive (or configural) reasoning (in this paper this was exemplified as encouraging reasoning based on type $2 \mathrm{~A}, 2 \mathrm{~B}$ or $2 \mathrm{C}$ to type 3 ), which is an answer to our RQ2.

\section{Conclusion and limitation of the study}

We consider that our findings are significant as this is one of the first studies to use the same survey questions to see how students in each grade perform certain geometry problem solving, and what kind of difficulties they show. In this paper, we mainly relied on students' written evidence and thinking processes observed in one lesson, but our approach has certain limitations. Amongst the limitations, our data and approach is not enough to scrutinise why and how students chose particular spatial reasoning skills and domain-specific knowledge. Such metacognitive aspects are missing in this paper and are worthy of consideration in future research.

While we have deepened insight into the use of spatial reasoning skills in geometry across the grades, our findings are based on the survey questions used in this study. Taking a factor analysis approach, as Pittalis and Christou (2010) did in constructing a model for specific and general spatial reasoning skills with a wider range of different question items, is something to consider for future research. Gaining further insights into how the spatial reasoning skills revealed in our study might be related to other areas of mathematics such as proving or algebraic reasoning is also something we would like to undertake in our future research.

Open Access This article is licensed under a Creative Commons Attribution 4.0 International License, which permits use, sharing, adaptation, distribution and reproduction in any medium or format, as long as you give appropriate credit to the original author(s) and the source, provide a link to the Creative Commons licence, and indicate if changes were made. The images or other third party material in this article are included in the article's Creative Commons licence, unless indicated otherwise in a credit line to the material. If material is not included in the article's Creative Commons licence and your intended use is not permitted by statutory regulation or exceeds the permitted use, you will need to obtain permission directly from the copyright holder. To view a copy of this licence, visit http://creativecommons.org/licenses/by/4.0/.

\section{References}

Aspinwall, L., Shaw, K. L., \& Presmeg, N. C. (1997). Uncontrollable mental imagery: graphical connections between a function and its derivative. Educational Studies in Mathematics, 33(3), 301-317. 
Battista, M. T., Frazee, L. M., \& Winer, M. L. (2018). Analyzing the relation between spatial and geometric reasoning for elementary and middle school students. In K. S. Mix \& M. T. Battista (Eds.), Visualizing Mathematics: the role of spatial reasoning in mathematical thought (pp. 195-228). Cham: Springer.

Chinnappan, M., Ekanayake, M. B., \& Brown, C. (2012). Knowledge use in the construction of geometry proof by Sri Lankan students. International Journal of Science and Mathematics Education, 10(4), 865887.

Duval, R. (2017). In M. Tania \& M. Campos (Eds.), Understanding the mathematical way of thinking: the registers of semiotic representations. Cham: Springer.

Fischbein, E. (1993). The theory of figural concepts. Educational Studies in Mathematics, 24(2), 139-162.

Fujita, T. (2012). Learners' level of understanding of the inclusion relations of quadrilaterals and prototype phenomenon. The Journal of Mathematical Behavior, 31(1), 60-72.

Fujita, T., Kondo, Y., Kumakura, H., \& Kunimune, S. (2017). Students' geometric thinking with cube representations: assessment framework and empirical evidence. The Journal of Mathematical Behavior, 46, 96-111.

Gillard, E., Van Dooren, W., Schaeken, W., \& Verschaffel, L. (2009). Dual processes in psychology of mathematics education and cognitive psychology. Human Development, 52, 95-108.

Jones, K., \& Tzekaki, M. (2016). Research on the teaching and learning of geometry. In A. Gutiérrez, G. C. Leder, \& P. Boero (Eds.), The second handbook of research on the psychology of mathematics education (pp. 109-149). Rotterdam: Sense Publishers.

Kondo, Y., Fujita, T., Kunimune, S., Jones, K., \& Kumakura, H. (2014). The influence of 3D representations on students' level of 3D geometrical thinking. In Proceedings of PME 38 and PME-NA 36 (Vol. 4, pp. 25-32).

Llinares, S., \& Clemente, F. (2019). Characteristics of the shifts from configural reasoning to deductive reasoning in geometry. Mathematics Education Research Journal, 31(3), 259-277.

Lowrie, T. (2012). Visual and spatial reasoning: the changing form of mathematics representation and communication. In B. Kaur \& T. L. Toh (Eds.), Reasoning, communication and connections in mathematics: Yearbook 2012, Association of Mathematics Educators (pp. 149-168). Singapore: World Scientific.

Lowrie, T., Logan, T., Harris, D., \& Hegarty, M. (2018). The impact of an intervention program on students' spatial reasoning: Student engagement through mathematics-enhanced learning activities. Cognitive Research: Principles and Implications, 3(50), 1-10.

Lowrie, T., Logan, T., \& Hegarty, M. (2019). The influence of spatial visualization training on students' spatial reasoning and mathematics performance. Journal of Cognition and Development, 20(5), 729-751.

Mulligan, J., Woolcott, G., Mitchelmore, M., \& Davis, B. (2018). Connecting mathematics learning through spatial reasoning. Mathematics Education Research Journal, 30(1), 77-87.

Parzysz, B. (1991). Representation of space and students' conceptions at high school level. Educational Studies in Mathematics, 22(6), 575-593.

Pittalis, M., \& Christou, C. (2010). Types of reasoning in 3D geometry thinking and their relation with spatial ability. Educational Studies in Mathematics, 75(2), 191-212.

Pittalis, M., \& Christou, C. (2013). Coding and decoding representations of 3D shapes. The Journal of Mathematical Behavior, 32(3), 673-689.

Sijtsma, K., \& Molenaar, I. W. (2002). Introduction to nonparametric item response theory (Vol. 5). Thousand Oaks: Sage.

Sinclair, N., \& Bruce, C. D. (2015). New opportunities in geometry education at the primary school. ZDM Mathematics Education, 47(3), 319-329.

Sinclair, N., Bussi, M. G. B., de Villiers, M., Jones, K., Kortenkamp, U., Leung, A., \& Owens, K. (2016). Recent research on geometry education: An ICME-13 survey team report. ZDM, 48(5), 691-719.

Ufer, S., Heinze, A., \& Reiss, K. (2008). Individual predictors of geometrical proof competence. Proceedings of the Joint Meeting of PME 32and PME-NA XXX (Vol. 4, pp. 361-368).

Van Hoof, J., Lijnen, T., Verschaffel, L., \& Van Dooren, W. (2013). Are secondary school students still hampered by the natural number bias? A reaction time study on fraction comparison tasks. Research in Mathematics Education, 15(2), 154-164.

Publisher's note Springer Nature remains neutral with regard to jurisdictional claims in published maps and institutional affiliations. 